\title{
Posterior Parietal Cortex Guides Visual Decisions in Rats
}

\author{
Angela M. Licata, ${ }^{1}$ Matthew T. Kaufman, ${ }^{1}$ David Raposo, ${ }^{1,2}$ Michael B. Ryan, ${ }^{3}{ }^{\oplus}$ John P. Sheppard, ${ }^{4,5}$ \\ and ${ }^{D}$ Anne K. Churchland ${ }^{1}$ \\ ${ }^{1}$ Cold Spring Harbor Laboratory, Cold Spring Harbor, New York 11724, ${ }^{2}$ Champalimaud Neuroscience Programme, Lisboa, Portugal 1400-038, ${ }^{3}$ University \\ of California, San Francisco, California 94143, ${ }^{4}$ University of California, Los Angeles, California 90095, and ${ }^{5}$ Northwestern University, Chicago, Illinois 60208
}

Neurons in putative decision-making structures can reflect both sensory and decision signals, making their causal role in decisions unclear. Here, we tested whether rat posterior parietal cortex (PPC) is causal for processing visual sensory signals or instead for accumulating evidence for decision alternatives. We disrupted PPC activity optogenetically during decision making and compared effects on decisions guided by auditory versus visual evidence. Deficits were largely restricted to visual decisions. To further test for visual dominance in PPC, we evaluated electrophysiological responses after individual sensory events and observed much larger response modulation after visual stimuli than auditory stimuli. Finally, we measured trial-to-trial spike count variability during stimulus presentation and decision formation. Variability decreased sharply, suggesting that the network is stabilized by inputs, unlike what would be expected if sensory signals were locally accumulated. Our findings suggest that PPC plays a causal role in processing visual signals that are accumulated elsewhere.

Key words: decision making

\section{Significance Statement}

Defining the neural circuits that support decision making bridges a gap between our understanding of simple sensorimotor reflexes and our understanding of truly complex behavior. However, identifying brain areas that play a causal role in decision making has proved challenging. We tested the causal role of a candidate component of decision circuits, the rat posterior parietal cortex (PPC). Our interpretation of the data benefited from our use of animals trained to make decisions guided by either visual or auditory evidence. Our results suggest that PPC plays a causal role specifically in visual decision making and may support sensory aspects of the decision, such as interpreting the visual signals so that evidence for a decision can be accumulated elsewhere.

\section{Introduction}

A large body of work has documented neural responses during perceptual decisions (Roitman and Shadlen, 2002; Churchland et al., 2008; Ding and Gold, 2012; Rishel et al., 2013; Ding, 2015; Hanks et al., 2015). These studies reveal cortical and subcortical structures that might constitute a brain-wide circuit for transforming raw sensory inputs into plans for action. Although transient disruption of activity in these structures could help in assessing their causal role in such a circuit, these types of experi-

Received Jan. 12, 2017; revised March 23, 2017; accepted April 2, 2017.

Author contributions: A.K.C. designed research; A.M.L., D.R., M.B.R., and J.P.S. performed research; A.M.L., M.T.K., D.R., and A.K.C. analyzed data; A.M.L., M.T.K., and A.K.C. wrote the paper.

This work was supported by the National Institutes of Health (Grant EY022979 to A.K.C.), the McKnight Foundation (A.K.C.), the Simons Collaboration on the Global Brain (A.K.C., M.T.K.), the Pew Charitable Trusts (A.K.C.), the Klingenstein Foundation (A.K.C.), and the Marie Robertson Memorial Fund of Cold Spring Harbor Laboratory (A.K.C.). We thank Barry Burbach and Simon Musall for advice on the figures and analyses and Bernardo Sabatini for providing comments on a draft of the paper.

The authors declare no competing financial interests.

Correspondence and requests for materials should be addressed to Prof. Anne Churchland, Cold Spring Harbor Laboratory, 1 Bungtown Road, Cold Spring Harbor, New York 11724-2209. E-mail: churchland@cshl.edu.

DOI:10.1523/JNEUROSCI.0105-17.2017

Copyright $\odot 2017$ the authors $\quad 0270-6474 / 17 / 374954-13 \$ 15.00 / 0$ ments have been performed rarely and inconclusively. The importance of causal manipulations is underscored by experiments that found no effect of neural disruption on some decisions, even for areas in which neurons reflect decision signals (Suzuki and Gottlieb, 2013; Erlich et al., 2015; Chen et al., 2016; Katz et al., 2016).

The role of one candidate area, the posterior parietal cortex (PPC) of rodents, remains particularly ambiguous because existing work paints conflicting pictures of its role in decision making. Electrophysiological observations demonstrate that PPC is modulated during both auditory (Raposo et al., 2014; Hanks et al., 2015) and visual (Harvey et al., 2012; Raposo et al., 2014) decisions, which unfold gradually over $\sim 1 \mathrm{~s}$. These slow-time-course signals could reflect evidence accumulation or action planning either in PPC or in a remote area with feedback projections to PPC. One possibility is that evidence accumulation occurs remotely and PPC plays a predominately sensory role. Certainly, in order for sensory evidence to be accumulated, preliminary computations are required to extract the relevant signals from the overall sensory response. For example, if individual events need to be accumulated, then the brain must first detect and distinguish them. 
The ability of individual auditory events to drive PPC neurons has been noted as weak (Hanks et al., 2015), but this has not been studied in depth because recent work has focused on slower modulation over the course of the entire decision. Deficits for visual, but not auditory, decisions observed after PPC inactivation hint at a putative role in discriminating visual events (Raposo et al., 2014). However, these inactivation studies are not entirely conclusive because neural activity was suppressed continuously for 2-3 h. This leaves open the possibility that a role in evidence accumulation, or a role in detecting auditory events, might have been missed: $2-3 \mathrm{~h}$ of suppression might permit the animal to adjust its strategy, potentially recruiting alternate neural circuits that are not typically involved. Further, existing studies have not fully characterized the nature of the deficits to visual decisions, leaving it unclear whether inactivation affected sensory processing specifically or instead affected other decision factors.

Here, we examined PPC's contribution to decision making by manipulating and more closely measuring neural responses. First, we used a temporally precise optogenetic perturbation method to disrupt neural activity. By disrupting activity during both visual and auditory decisions, we found specific sensory processing effects; however, there was little in the way of more general effects on decisions, such as accumulation of evidence or the ability to report choices. A probabilistic decision analysis offered insight into effects of PPC disruption on nonsensory factors that guide decisions, such as a reliance on reward history. Second, we conducted a temporally precise analysis of previously collected electrophysiological data to isolate the impact of individual auditory and visual events on PPC responses, providing an independent and novel assessment of PPC's role in auditory and visual processing during decision making. Finally, we leveraged an analysis of trial-to-trial variability that is informative about the underlying computations taking place within an area. All three approaches support the same conclusion: PPC's contribution to perceptual decisions on this task is primarily sensory and may support computations related to detecting and distinguishing individual stimulus events. Higher-level choice or action-planning signals that can be observed in responses of PPC neurons likely reflect computations that take place elsewhere in the brain.

\section{Materials and Methods}

\section{Animal subjects}

Adult male Long-Evans rats (200-250 g; Taconic Farms) were housed with ad libitum access to food and restricted access to water starting from the onset of behavioral training. Rats were housed on a standard (nonreversed) light/dark cycle; experiments were run during the light part of the cycle. Rats were pair housed initially, but were singly housed once they received injections or implants (see below). All experimental procedures were in accordance with the National Institutes of Health's Guide for the Care and Use of Laboratory Animals and were approved by the Cold Spring Harbor Animal Care and Use Committee.

\section{Behavior}

Four freely moving rats were trained on a rate discrimination task (see Fig. 1A) as described previously (Raposo et al., 2012; Sheppard et al., 2013). Briefly, rats were trained to judge whether the overall rate of a repeating auditory (click) or visual (flash) stimulus was high or low compared with a learned category boundary (12.5 events/s, range 9-16 events/s). Click and flash events were separated by either a long $(100 \mathrm{~ms})$ or short $(50 \mathrm{~ms})$ interval. Trials with intermediate rates thus contained mixtures of long and short intervals, generating instantaneous rates that fluctuated over the course of $1000 \mathrm{~ms}$, during which time animals were required to keep their snout in a central port. A fixed duration of $1000 \mathrm{~ms}$ was selected to allow a more direct comparison with other studies of the PPC's role in the accumulation of evidence tasks that likewise used a fixed duration (Erlich et al., 2015; Katz et al., 2016). Rate fluctuations during this $1000 \mathrm{~ms}$ period encourage animals to use information throughout the trial to guide their decision. We have demonstrated previously that, for this stimulus, decisions are influenced by evidence presented over the majority of the $1000 \mathrm{~ms}$ period, consistent with accumulation of evidence during that time (Raposo et al., 2012; Sheppard et al., 2013).

After the $1000 \mathrm{~ms}$ period ended, rats indicated their decision on that trial by moving to a left or right reward port. Response time is defined as the time between when the stimulus ended and when the rat departed the port. Movement time is defined as the time between exiting the center port and entering a reward port. Movements to the correct reward port yielded a drop of water $(10-25 \mu \mathrm{l})$. Three of the four rats were trained that rightward choices were rewarded after high-rate stimuli and leftward choices were rewarded after low-rate stimuli; one rat was trained with the opposite contingency.

Animal training typically lasted 5-6 weeks and was completed before implanting the stimulation/recording assembly (see "Viral injection" and "Implants for electrophysiology" sections below).

\section{General surgical procedures}

All rats subjected to surgery were anesthetized with isoflurane and administered $5 \mathrm{mg} / \mathrm{kg}$ ketoprofen before surgery for analgesia. Isoflurane anesthesia was maintained by monitoring respiration and foot-pinch responses throughout the surgical procedure. Ophthalmic ointment was applied to keep the eyes moistened throughout surgery. Lidocaine solution $(\sim 0.1 \mathrm{ml})$ was injected below the scalp to provide local analgesia before performing scalp incisions. $0.05 \mathrm{mg} / \mathrm{kg}$ buprenorphine was administered daily for postsurgery analgesia (usually $2-3 \mathrm{~d}$ ).

\section{Viral injection}

Channelrhodopsin-2 (ChR2) expression was induced in the left PPC of three rats using adeno-associated virus (AAV, serotype 9) carrying the gene for ChR2 fused with green fluorescent protein (GFP) under the control of the CAG promoter (AAV9-CAG-ChR2-GFP). This promoter induces the expression of $\mathrm{ChR} 2$ in all cell types. Unilateral injections of this construct were made in the left PPC of 4- to 6-week-old rats. Two to three separate penetrations along the medial-lateral axis were made with the goal of maximizing expression in PPC and minimizing the spread outside of this area. Stereotactic coordinates (relative to bregma) for Rats 1 and 2 were $-3.8 \mathrm{~mm} \mathrm{AP,} \mathrm{2.2/3.2/4.2} \mathrm{mm} \mathrm{ML;} \mathrm{for} \mathrm{Rat} \mathrm{3,} \mathrm{they} \mathrm{were}-3.8$ $\mathrm{mm} \mathrm{AP}$ and 2.2/3.7 mm ML. A small craniotomy was made and a calibrated glass pipette positioned within the craniotomy perpendicular to the brain's surface. For the injection, pressure was applied to a syringe that was attached to the pipette via plastic tubing. Injections were made at 400, 600, and $800 \mu \mathrm{m}$ below the pial surface. At each depth, $140 \mathrm{nl}$ was injected. We refrained from deeper injections to avoid viral spread to subcortical structures.

Note that, because all injections were in the left hemisphere, lateralized effects are referred to as "ipsi" or "contra" because these mean the same for all animals. Because we trained different animals to associate the left versus right port with low rate choices, all behavioral data are plotted relative to the injected hemisphere. This convention makes it possible to distinguish biases toward a particular side from biases toward a particular rate because "high-rate" trials are not always associated with the same side.

\section{Implants for electrophysiology}

Rats were implanted with custom optetrode implants (Anikeeva et al., 2011) that were prepared in house. Each assembly contained up to eight independently moveable tetrodes (nickel/chrome alloy wire, $12.7 \mu \mathrm{m}$; Sandvik Kanthal). Tetrodes were connected to an EIB-36 narrow connector board (Neuralynx) mounted on the implant assembly. Six to eight of the tetrodes were attached to optical fibers used for delivering light (Anikeeva et al., 2011; Znamenskiy and Zador, 2013). Optical fibers were $62.5 \mu \mathrm{m}$ in diameter with a $50 \mu \mathrm{m}$ core. Each optical fiber was glued to a tetrode; the pair was mounted on an independently moveable microdrive. The assembly was secured within a plastic enclosure before implanting. Tetrodes were gold plated to $300-700 \mathrm{k} \Omega$ at $1 \mathrm{kHz}$; one additional tetrode was used as an internal reference for electrophysiological recordings and plated to $\sim 100 \mathrm{k} \Omega$. 
For implantation during surgery, we followed procedures described previously (Raposo et al., 2014). Briefly, we positioned the entire optetrode assembly so as to center it relative to the previously made injections $(-3.8 \mathrm{~mm} \mathrm{AP}$ and $2.5 \mathrm{~mm} \mathrm{ML})$. A durotomy was performed and the implant assembly was lowered until the tetrodes just penetrated the pial surface. Next, $2 \%$ agarose solution was applied to cover the tetrodes and craniotomy and dental acrylic (Lang Dental) was applied to secure the implant to the skull. The incision was closed around the base of the implant using 1-2 Vicryl sutures anterior and posterior to the implant. After surgery, tetrodes were advanced in increments of $40-80 \mu \mathrm{m}$ until action potentials were encountered. In all animals, we confirmed that stimulation elicited a clear change in the LFP on at least one tetrode, although responses were typically observed on multiple tetrodes. An example LFP is shown in Figure $1 C$ to demonstrate that we were able to observe clear changes in LFP after stimulation.

\section{Optogenetic stimulation}

We used blue light $(473 \mathrm{~nm})$ with intensity ranging from 5 to $20 \mathrm{~mW}$ at the fiber tip. To estimate the spread of light, we used a well established method, the brain light tissue transmitter, which estimates light spread based on wavelength, fiber diameter, numerical aperture, and power. We elected to use this method for three reasons. First, the estimates of light spread are accurate and reliable because of the systematic way in which the measurements are collected to generate the calculator (multiple measurements at each of many distances from fiber tip). Second, the estimates from the calculator are consistent with many additional published measurements for blue light spread in rodents, both in vivo (Guo et al., 2014) and in slice (Aravanis et al., 2007; Huber et al., 2008). The measurements in slice afford a very precise estimate because experimenters can measure light spread directly by placing the slice over the photodetector of a power meter. Finally, the use of published measurements is justified because the spread of light is likely to be homogeneous across animals; light spread mainly depends on the properties of brain tissue. As a result, judging the extent of stimulation is more straightforward compared with judging the spread of chemogenetic disruption. In chemogenetic disruption (Rogan and Roth, 2011), infected neurons are activated with equal probability by the ligand to a synthetic receptor delivered by a virus. Therefore, the extent of stimulation is determined mainly by the extent of viral spread, so quantifying the spread is essential. Indeed, we estimated spread of effect previously (Raposo et al., 2014). In the current study, extent of expression is less informative because neurons expressing ChR2 that are beyond the range of the blue light will be unaffected. Even if we had expressed ChR2 nonspecifically across the brain, we still would have achieved specificity because of the restriction of the blue light. Indeed, studies routinely achieve specificity with blue light stimulation in animals expressing ChR2 brain wide (Guo et al., 2014).

Based on the diameter of the fiber $(50 \mu \mathrm{m})$ and its numerical aperture (0.22), we estimate (http://web.stanford.edu/group/dlab/optogenetics/) that, at a distance of $0.5 \mathrm{~mm}$ away from the fiber, irradiance was 24.8 $\mathrm{mW} / \mathrm{mm}^{2}$ and, at a distance of $1.15 \mathrm{~mm}$ away, it was $<0.5 \mathrm{~mW} / \mathrm{mm}^{2}$. Given that $0.5 \mathrm{~mW} / \mathrm{mm}^{2}$ has been shown to be the minimal required intensity to induce spiking in awake animals (Guo et al., 2014), we infer that our stimulation mainly affected ChR2-expressing neurons within this range. One possibility that these calculations make unlikely is that the blue light (and thus the direct activation) spread to primary visual cortex (V1). Our stimulating fiber was positioned at $3.8 \mathrm{~mm}$ posterior to bregma. A distance of $1.15 \mathrm{~mm}$ away from the fiber (where light is too weak to drive neurons; see above) corresponds to $\sim 4.9 \mathrm{~mm}$ posterior to bregma. There, the very most anterior tip of $\mathrm{V} 1$ is $1.5 \mathrm{~mm}$ lateral to where we positioned our optetrode (Paxinos and Watson, 2007). Even if a small number of V1 neurons were somehow affected, our full-field stimulus would only have altered the response of a few $\mathrm{V} 1$ neurons representing the extreme lower nasal edge of one hemifield. Activation of V1 neurons is therefore very unlikely to be responsible for our behavioral effects.

On a subset of randomly selected trials ("stimulation trials," 15-35\%), we delivered blue light to activate ChR2-expressing neurons in PPC using a 473 nanometer diode-pumped solid-state laser. On these trials, the laser was triggered at the beginning of the stimulus presentation (visual or auditory) and was kept on throughout the entire decision formation period (1000 ms), delivering light pulses at a rate of $42 \mathrm{~Hz}$ (Fig. 1D). On the remaining trials ("control trials"), no optical stimulation occurred. We used two techniques to minimize the rats' ability to detect the optical trials by seeing the blue light. In Rats 1 and 2, we covered the implant with black insulating tape before beginning each session. To ensure that no light was emitted, the experimenter would deliver light into the laser while it was connected to the animal in a dark booth and visually inspect the implant for any escaping light. A second method was developed because adding and removing tape from the implant daily reduced the integrity of the implant and sometimes resulted in premature explantation. In this second method, the implant was not covered in tape, but we also used a second optical fiber in a ferrule not implanted in the brain (i.e., light from this laser was blocked from entering the brain). Light from this second fiber was still visible and thus served to mask the light from the stimulation. The second fiber was illuminated on every trial (control trials and stimulation trials). As a result, the presence of blue light would be difficult to use to detect optical stimulation trials.

We typically collected data from a single "site" (stimulation on one fiber at a particular depth) for 5-8 d; behavioral data were pooled over those days. We then either stimulated on a different tetrode or advanced the current optetrode at least $200 \mu \mathrm{m}$. We first assessed the effects of stimulation on the rat's overall proportion of correct choices (see Fig. 3) and then analyzed stimulation effects in more detail.

\section{Analysis of stimulation effects}

We measured the effects of stimulation on three aspects of behavior. First, we used a probabilistic decision model (Busse et al., 2011) to determine systematically the effects of stimulation on four factors contributing to the animal's decision. The rat's binary choice $Y$ on each trial is given by the following:

$$
\ln \left(\frac{p}{1-p}\right)=\beta_{0}+\beta_{1} r+\beta_{2} h_{\text {success }}+\beta_{3} h_{\text {fail }}
$$

where $p=P(Y=1 \mid \beta, r, h), r$ is the stimulus strength (its rate relative to the $12.5 \mathrm{~Hz}$ category boundary; the true range of -3.5 to 3.5 events/s above and below the boundary were scaled so that values ranged from -1 to 1$), h_{\text {success }}$ indicates whether the previous trial was a success ( 1 if the contralateral side was rewarded, -1 if the ipsilateral side was rewarded; 0 otherwise), and $h_{\text {fail }}$ indicates whether the previous trial was a failure ( 1 if the failure followed a decision to the contralateral side, -1 if the failure followed a decision to the ipsilateral side; 0 otherwise). The coefficients were fit in MATLAB (The MathWorks) using glmfit and a logit linking function. The observer's decision was predicted as a combination of four factors, so the values of the fitted coefficients $\left(\beta_{0-3}\right)$ provide insight into how much each parameter of the model influences the decision on any given trial. Stimulation (laser-on) and control (laser-off) trials were fitted separately so that the coefficients could be compared. In principle, only the rate $(r)$ should influence the rat's decision because this determines the reward contingency; however, previous work has shown that, in practice, side bias and reward history bias are influential. If animals were to rely more on reward history bias on stimulation trials, then this would have reduced their overall performance because the correct response for the current trial is independent of the previous trial. Therefore, this analysis afforded a deeper insight into the factors that could alter the rat's accuracy on stimulation trials.

To assess significance of differences in the fitted coefficients (see Fig. 4), two tests were performed. For the first test, we conducted one-sided paired $t$ tests for each site separately. The effect of stimulation was evaluated for each of the four fitted parameters: bias, sensitivity, success history, and failure history. The $t$-statistic for each was computed directly using the values of the fitted parameters and their associated SE returned by glmfit (computed from the square root of the diagonal values of the covariance matrix). The SE on the difference was calculated by propagating the error associated with the stimulation and no-stimulation values of each parameter. Second, we conducted one-sided paired $t$ tests for the data pooled across all sites and animals.

For the second test, we measured the effect of stimulation on key temporal aspects of the behavioral response. For instance, we determined 
Table 1. Movement metrics computed separately for each animal, pooled across all sites tested

\begin{tabular}{|c|c|c|c|c|c|c|c|c|c|c|c|c|}
\hline & \multicolumn{4}{|c|}{ Prop. early withdrawal } & \multicolumn{4}{|c|}{ Response time (ms) } & \multicolumn{4}{|c|}{ Movement duration (ms) } \\
\hline & Control & Stim & $\mathrm{p}$ & $\mathrm{N}$ & Control & Stim & $\mathrm{p}$ & $\mathrm{N}$ & Control & Stim & $\mathrm{p}$ & $\mathrm{N}$ \\
\hline \multicolumn{13}{|c|}{ Visual trials } \\
\hline Rat 1 & 0.254 & 0.194 & $<10-3$ & 3622 & 157.7 & 153.0 & 0.188 & 2796 & 542.5 & 540.0 & 0.584 & 2796 \\
\hline Rat 2 & 0.237 & 0.264 & 0.015 & 7222 & 219.9 & 212.2 & 0.045 & 5430 & 592.7 & 597.3 & 0.281 & 5430 \\
\hline Rat 3 & 0.344 & 0.296 & $<10-3$ & 17837 & 214.9 & 207.4 & 0.010 & 11757 & 490.2 & 485.1 & 0.054 & 11757 \\
\hline \multicolumn{13}{|c|}{ Auditory trials } \\
\hline Rat 1 & 0.191 & 0.101 & $<10-3$ & 2723 & 176.3 & 180.5 & 0.341 & 2313 & 536.8 & 523.5 & 0.007 & 2313 \\
\hline Rat 2 & 0.208 & 0.214 & 0.518 & 7406 & 262.0 & 241.4 & $<10-3$ & 5823 & 602.6 & 604.1 & 0.708 & 5823 \\
\hline Rat 3 & 0.299 & 0.235 & $<10-3$ & 8150 & 216.6 & 212.5 & 0.389 & 5729 & 480.0 & 491.3 & 0.006 & 5729 \\
\hline
\end{tabular}

whether stimulation changed the animal's ability to remain in the center port for the required $1000 \mathrm{~ms}$ duration. Specifically, we evaluated whether the proportion of trials in which the animal withdrew early differed for stimulation versus control trials $\left(\chi^{2}\right.$ test; Table 1$)$. Further, we evaluated the time that elapsed between when the stimulus ended and when the animal exited the center port (response time). We used an unpaired, two-sided $t$ test to evaluate whether response times differed for stimulation and control trials (see Fig. 3B, Table 1). Finally, we evaluated the time that elapsed between when the rat left the center port and when it arrived at one of the two side ports (movement duration). We used an unpaired, two-sided $t$ test to evaluate whether movement duration differed for stimulation and control trials (see Fig. 3C, Table 1).

We wished to estimate the window over which sensory stimuli influenced the rat's decision and to evaluate whether disruption affected that window. To achieve this, we used a regression analysis (Katz et al., 2016) that computed separately the influence of successive $100 \mathrm{~ms}$ time windows on the rat's eventual decision (see Fig. $4 D, J$ ). The weight of each time window was computed via logistic regression, where the rat's binary choice $Y$ on each trial is given by the following:

$$
\ln \left(\frac{p}{1-p}\right)=\beta_{0}+x^{T} w
$$

where $p=P\left(Y=1 \beta_{0}, x, w\right), \beta_{0}$ is a scalar bias term, $x$ is a vector of the 10 successive time windows over the trial, and $w$ is a vector of the weights for each time window. The weight vector $w$ was computed using glmfit (MATLAB). This analysis can reveal whether stimulation changed how the animal used information as it arrives over time, potentially revealing leaky accumulation or a tendency to make "snap judgments." The analysis is similar to the excess rate analysis that we have used previously (Raposo et al., 2012; Sheppard et al., 2013), but has the advantage of not requiring the exclusion of many trials. Data were pooled across the three injected animals to provide sufficient power to estimate weights on stimulation trials, which were rarer.

\section{Analysis of electrophysiological responses}

We performed two analyses of electrophysiological responses from a previously collected dataset (Raposo et al., 2014). We used this dataset instead of the electrophysiological dataset associated with optogenetic stimulation because of its large size ( $n=101,972$ trials). Although we did record well isolated neurons with the optetrodes used here (see Fig. 1D), the population size did not provide the statistical power needed for the relevant analyses. Because the previous dataset consists of rats performing an identical task, the data were ideally suited to these analyses.

Analysis of the effect of single stimulus events on neural responses. For the first analysis (see Fig. $8 B-D$ ), we wished to determine whether single flash or click events modulated firing rates at fast timescales independent of overall condition modulation (the tuning captured in a typical peristimulus time histogram). To do so, we considered all successful trials of the highest or lowest rates separately. We first smoothed each trial's firing rate with an acausal Gaussian ( $15 \mathrm{~ms} \mathrm{SD}$ ). We then made perievent time histograms (PETHs) for low-rate trials. The first three events of every trial were discarded to reduce the effects of onset transients and adaptation. Intuitively, if neurons were modulated by individual events, then we would expect quick deflections in the firing rate after each flash or click.
If a neuron exhibited choice tuning (a slow upward or downward ramp in firing rate over the course of the whole stimulus), then this would appear in the PETH as a near-linear trend. To remove these trends, we linearly detrended each PETH using 1.5 cycles of the stimulus. Next, as a simple raw estimate of event modulation $\left(m_{\text {raw }}\right)$, we computed the SD of this PETH to capture whatever deflection was present. However, noise always has a nonzero SD, so this estimate is biased: noisier neurons will tend to produce higher values of $m_{\text {raw }}$. To correct for this bias, we assumed that the observed spiking was the sum of the "true" underlying fluctuations in the firing rate (event tuning) plus noise (Shadlen and Newsome, 1998; Nawrot et al., 2008; Churchland et al., 2011). The variance of the noise process at any time point is simply the square of the SEM (denoted $\sigma_{\text {mean }}{ }^{2}$ ). Because event modulation was typically small compared with total firing rate, we approximated the total noise by averaging the SEM over time points. Because variances add for the sum of independent random processes, $m_{\text {raw }}^{2}=m^{2}+{\overline{\sigma_{\text {mean }}}}^{2}$ and our modulation index was therefore:

$$
m=\sqrt{m_{\text {raw }}^{2}-{\overline{\sigma_{\text {mean }}}}^{2}}
$$

For neurons where $m_{\text {raw }}^{2}<{\overline{\sigma_{\text {mean }}}}^{2}$, we assigned $m=0$. To obtain the modulation index for high-rate trials, the same process was repeated using events from high-rate trials. Note that values of exactly 0 were excluded from the histograms in Figure $8 D$.

To assess significance for each neuron, we wished to know how often pure noise (true $m=0$ ) would give rise to a modulation index as large as what we observed due to chance. Our modulation index is essentially just a SD. For a Gaussian random process, the SE of a SD is as follows: $S E(\sigma)=\sigma^{2} \sqrt{2 / D O F}$, where DOF is the number of degrees of freedom and here $\sigma=\overline{\sigma_{\text {mean }}}$. To estimate the DOF of the smoothed series, we used the common approximation $\operatorname{Tr}\left(S_{\lambda}\right)$, where $S_{\lambda}$ is the linear smoothing matrix and $\operatorname{Tr}(-)$ indicates taking the trace of a matrix. Using this approximation, for a Gaussian smoothing kernel, the equation is as follows:

$$
D O F \approx \frac{N}{\sigma_{\text {smoothing }} \sqrt{2 \pi}}
$$

For each neuron, we then converted $m$ and $S E\left(\overline{\sigma_{\text {mean }}}\right)$ into a one-tailed $p$-value. By inspection of individual neurons, event modulation was almost always greater for low-rate trials, so only low-rate trials were tested for significance. These $p$-values were not corrected for multiple comparisons because the goal was to obtain an estimate of the number of modulated neurons, not to determine whether any neurons were modulated. We also computed a Bonferroni-corrected $p$-value threshold for the latter purpose: at a significance level of $p<0.05 / 317,45$ neurons were significant for visual and 2 for auditory.

Analysis of trial-to-trial variance to provide insight on neural computation. To understand how trial-to-trial variability evolved over the course of auditory and visual decisions, we computed a measure of spike count variability, the variance of the conditional expectation (VarCE) (Churchland et al., 2011; Brostek et al., 2013; Marcos et al., 2013; Ding, 2015). Briefly, this measure assumes that the total measured spike count variance can be divided into two components using the law of total variance for doubly stochastic processes: (1) variance of counts that would be 
A

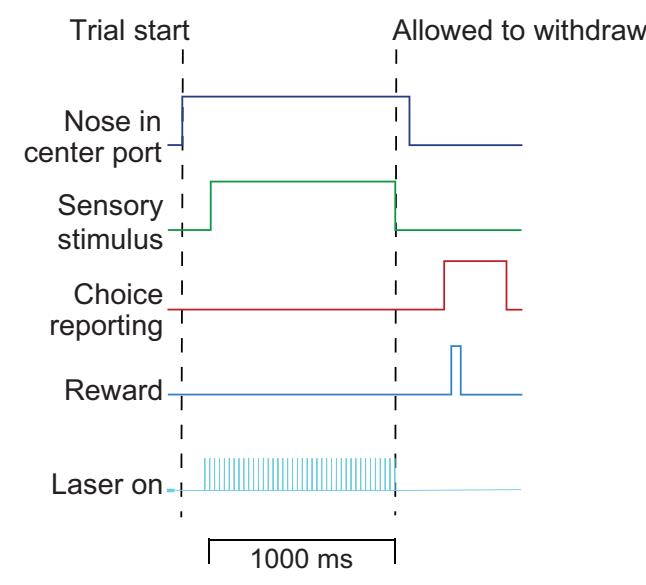

B

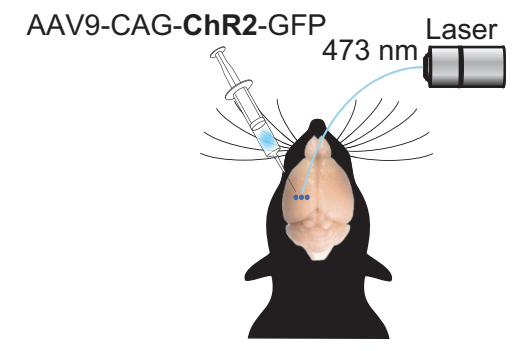

C

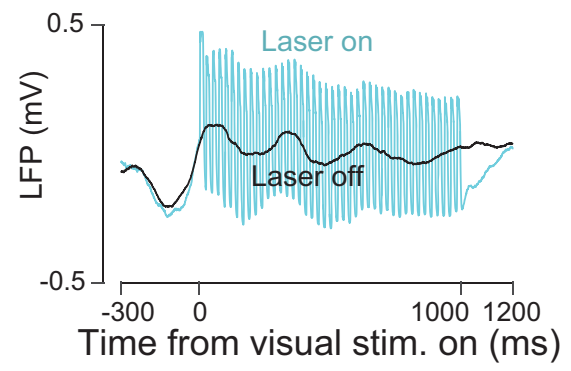

D

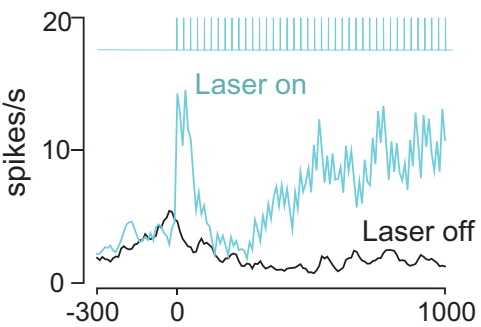

Time from visual stim. on (ms)

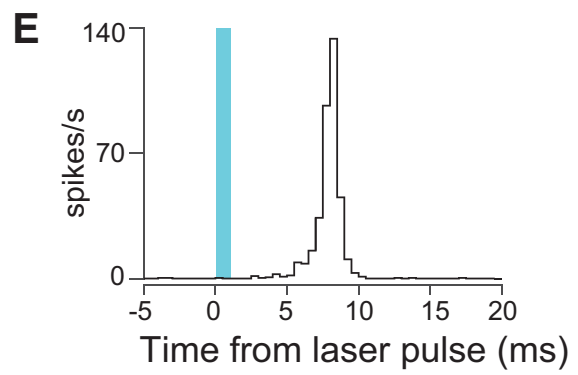

Figure 1. Decision-making task and strategy for disrupting PPC activity. $\boldsymbol{A}$, Schematic of decision-making task. Rats initiated trials by inserting their snouts into a port spanned by an infrared beam (dark blue trace). After a variable delay, a series of auditory or visual events began (green trace). Animals were required to remain in a center port for $1000 \mathrm{~ms}$ during which time these sensory stimuli were presented. Animals were then allowed to withdraw. They reported choices (red trace) at either a left or right decision port and were rewarded with a drop of water (light blue trace) when correct. Optogenetic stimulation ( $42 \mathrm{~Hz}, 5-20 \mathrm{~mW}$, cyan trace) was presented throughout the $1000 \mathrm{~ms}$ period on randomly selected trials. $\boldsymbol{B}$, Schematic of optogenetic approach showing unilateral injections of AAV9-CAG-ChR2-GFP into PPC. C, LFP recorded during laser-on and laser-off trials via a tetrode attached to the stimulating optical fiber. $\boldsymbol{D}$, Peristimulus time histogram for an example well isolated single neuron for laser-on (cyan) and laser-off (black) trials. E, PETH in which responses are aligned to individual pulses of blue light.

produced by a stochastic point process with a given rate ("spiking noise") and (2) the variance of the rates that would produce those counts ("conditional expectation"). The VarCE isolates the second of these components and is therefore informative about underlying mechanism. In principle, the VarCE is computed by subtracting an estimate of the first component from the total spike count variance as follows:

$$
S_{\left\langle N_{i}\right\rangle}^{2}=S_{N_{i}}^{2}-\phi \bar{N}_{i}
$$

where $N_{\mathrm{i}}$ is a vector of spike counts for a given neuron and given condition in time window $i, S_{N_{i}}^{2}$ is the sample variance of those spike counts, $\bar{N}_{i}$ is the mean spike count of a neuron across trials of a given condition in time window $i$, and $\phi$ is a constant that approximates the degree to which spike count variability scales with firing rate (Geisler and Albrecht, 1995; Nawrot et al., 2008). In practice, as in previous work, we computed $\phi$ separately for each neuron in the dataset by taking the minimum of the measured Fano factor across all conditions and time points. To make it possible to combine data from multiple conditions, we estimated $S_{N_{i}}^{2}$ using the residuals; that is, by subtracting from each sample count the mean for all trials sharing its condition. The VarCE plotted in Figure $8 E$ is the variance of the union of residuals from all conditions minus the weighted average of the stochastic variance $(\phi \bar{N})$ (see Eq. 6 in Churchland et al., 2011). A sliding time window with a width of $100 \mathrm{~ms}$ was used for the traces in Figure 8E. Longer $(150 \mathrm{~ms})$ and shorter $(60 \mathrm{~ms})$ windows yielded similar results.

\section{Results}

Optogenetic disruption of PPC reduces the accuracy of visual decision making

To probe for a causal role for PPC, we used a perceptual decision task (Raposo et al., 2012; Sheppard et al., 2013; Raposo et al., 2014) in which freely moving rats judge whether the fluctuating rate of a 1000 ms series of auditory or visual events (rate range: $9-16 \mathrm{~Hz})$ is high or low compared with an abstract category boundary (12.5 Hz; Fig. 1A).

Our strategy for disrupting PPC was to stimulate optogenetically PPC neurons expressing ChR2 (Boyden et al., 2005) in all cell types (Fig. 1B). This approach, disruption via elevation of responses of neurons nonspecifically, has been widely used (Churchland and Shenoy, 2007; Roberts et al., 2012; Rodgers and DeWeese, 2014; Otchy et al., 2015). It is effective in areas such as PPC in which neurons with heterogeneous response properties are spatially intermixed (Raposo et al., 2014). Short-latency changes could be observed in the LFP and the spikes of single units on the occasions that we were able to isolate them (Fig. $1 C-E$ ), confirming expression of ChR2. Further, histology obtained at the end of the experiment (Fig.

2) indicated robust virus expression in all three injected rats.

Stimulation reduced the animal's decision accuracy, a reduction that was large and significant on visual trials (Fig. $3 \mathrm{~A}$, left, 
A

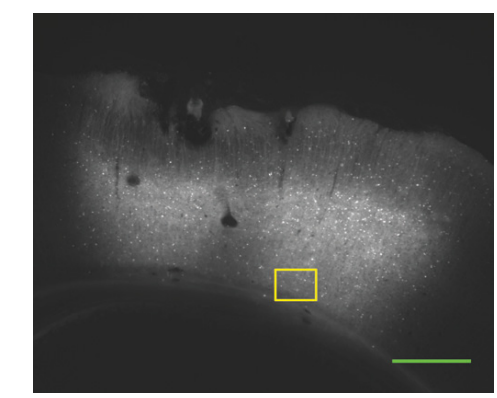

C

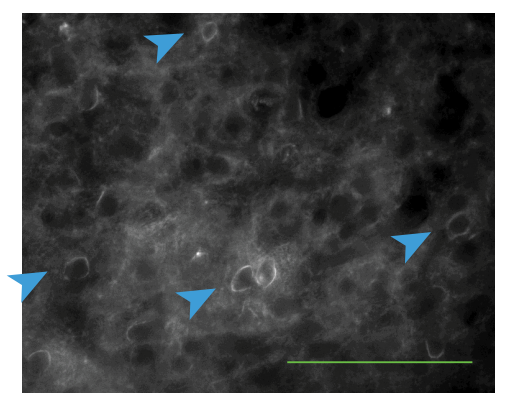

B

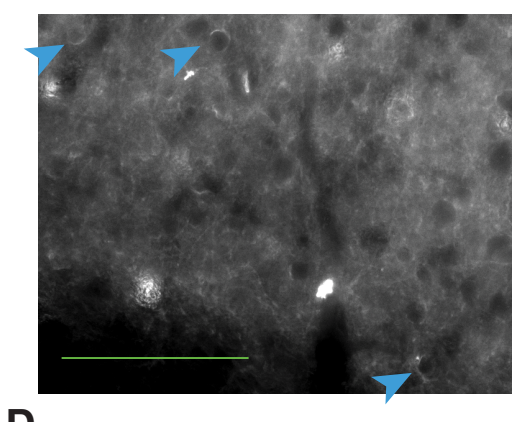

D

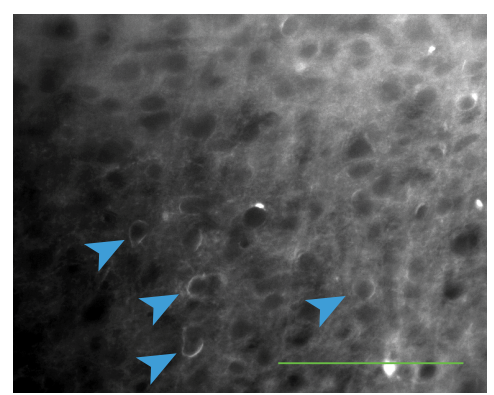

Figure 2. Robust expression of GFP-tagged ChR2 in PPC. $A$, Brain section from Rat 3 showing injection site within PPC. Green scale bar, $500 \mu \mathrm{m}$. Yellow box indicates region that will be magnified in the subsequent panel. $\boldsymbol{B}$, Further magnified view of the same image. Blue arrows indicate individual cells with membrane-bound GFP indicating the presence of ChR2. Green scale bar, 100 $\mu \mathrm{m} . \boldsymbol{C}, \boldsymbol{D}$, Same as $\boldsymbol{B}$ but for Rats 1 and 2.

$p=0.0002$ ) and modest and insignificant on auditory trials (Fig. $3 A$, right, $p=0.08$ ). The effect of stimulation on the proportion of correct trials was larger for visual than for auditory trials (2sided $t$ test, $p=0.01$ ). The larger effect on visual decisions was not due to a difference in baseline performance. Animals' accuracy on control trials was similar for auditory versus visual decisions: averaged across all stimulus difficulty levels, the proportion of correct choices was 0.68 correct for auditory trials versus 0.70 for visual trials, a difference that did not reach significance $(p=0.08$, paired $t$ test). The effects of stimulation were restricted to decision accuracy and were not overall detrimental to the animals' ability to engage in the task or report choices. The proportion of trials that were aborted because of an early withdrawal from the center port did not increase appreciably with stimulation and in two animals showed a significant decrease, providing reassurance that the stimulation did not cause the rats to become impulsive (Table 1). Stimulation also did not lead to sluggish movements: the time that it took for animals to withdraw from the center port once the stimulus had ended was similar on stimulation and control trials; in the significant cases, animals were in fact slightly faster on stimulation trials (response time; Fig. $3 B$, Table 1). Finally, the time that elapsed between when animals left the center port and when they arrived at a reward port was similar on stimulation and control trials (movement duration; Fig. 3C, Table 1).

\section{Visual accuracy deficits stem from a loss of sensitivity}

The reduction in visual accuracy that we observed could be due to disruption of any of a number of steps in the process by which the animal converts incoming sensory signals into a decision (Gold and Ding, 2013). To gain insight into which steps in the decision process were disrupted, we visualized the stimulation and control data as psychometric functions in which the proportion of cor- rect choices is plotted as a function of the stimulus rate (Fig. 4A). For visual trials at this example site, the psychometric function on stimulation trials (blue) is shallower than that for control trials (black). This means that a given change in visual stimulus rate (horizontal axis) had a weaker effect on decisions (vertical axis) for stimulation versus control trials, a change that contributed to the overall reduction in decision accuracy evident in Figure $3 A$ (left).

To quantify and understand these changes more deeply and to compare them across animals and stimulation sites, we used a probabilistic decision model (Lau and Glimcher, 2005; Busse et al., 2011) (see Materials and Methods). This model included sensitivity to stimulus rate, bias, and two additional factors that affect decisions (related to trial history; see below). Each factor could in principle be affected by stimulation, offering insight into the precise nature of the observed deficits. The most consistent factor affected by stimulation was reduced sensitivity, which was significant overall (Fig. $4 B$, most points below the $y=x$ line; $p=$ 0.0003; effects were individually significant in 7 of 11 sites; $p<0.01, t$ test). This loss of sensitivity serves to reduce the steepness of the psychometric function described above (Fig. 4A). To test whether this loss of sensitivity was temporally specific, we examined the sensitivity of control trials that immediately followed a stimulation trial. No effect on sensitivity for these trials was evident (data not shown, $p=0.49$ ). The restriction of sensitivity effects to the current trial confirms that our optogenetic strategy was successful in driving temporally precise disruption.

In addition to a loss of sensitivity, the shallower psychometric functions (and worse accuracy) on stimulation trials might be explained by an increased tendency for rats to be influenced by the previous trial's outcome. Because trials are generated independently, any influence of the previous trial, such as repeating a successful decision, is deleterious and would serve to reduce overall accuracy (Busse et al., 2011). The probabilistic decision analysis ruled out increased reliance on trial history as an explanation for deficits on stimulation trials. Stimulation had a very weak effect on the degree to which the current decision was influenced by the previous trial's success (Fig. $4 C, p=0.16$; 2 of 11 individual sites were significant, $p<0.01, t$ test) or failure (Fig. $4 E, p=0.04$, $t$ test, 0 of 11 individual sites were significant, $p<0.01, t$ test). These results rule out two "strategy" explanations for the stimulation effects, supporting the hypothesis that stimulation drove a loss of visual sensitivity.

The fourth parameter in the probabilistic decision model is the animal's bias. Bias is defined as a tendency for animals to favor one side over the other regardless of the strength of the sensory evidence. Under the hypothesis that PPC in one hemisphere is involved preferentially in computations relevant to the contralateral side (Crowne et al., 1986; Hanks et al., 2006), disrupting PPC in one hemisphere could bias the animal away from contralateral choices, driving an ipsilateral bias. We observed this ipsilateral bias at a number of sites (Fig. $4 F$, most points below the $y=x$ line; 

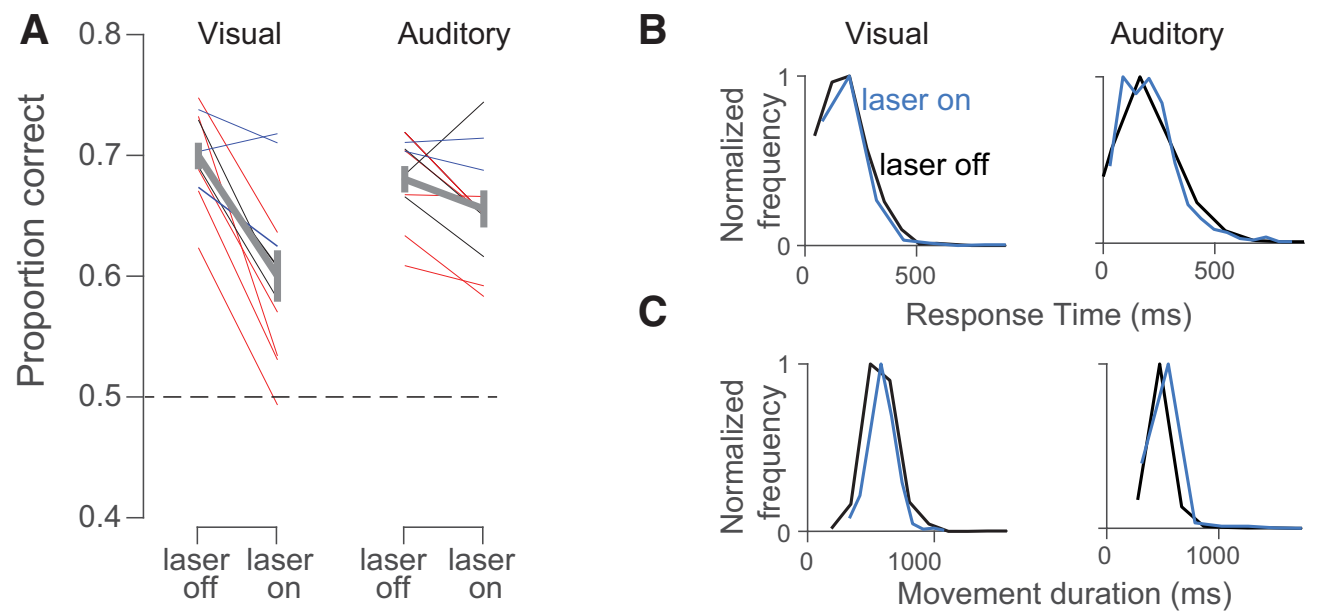

Figure 3. Stimulation drives a strong reduction in visual, but not auditory, decision accuracy and largely spares movements. $A$, Proportion correct for laser on versus laser off trials for visual (left) and auditory (right) trials. Each line illustrates values for a single site; lines of the same color are from the same animal ( 3 rats total). Thick gray line indicates mean ( \pm SEM) for all sites. Dashed line indicates chance performance. $\boldsymbol{B}$, Response times from an example site in one rat were similar after laser-on (blue) and laser-off (black) trials on both visual (left, 183 vs $184 \mathrm{~ms}, p=0.16$ ) and auditory (right, 209 vs $211 \mathrm{~ms}, p=0.67$ ) decisions. C, Movement durations from an example site were similar after laser-on (blue) and laser-off (black) trials on both visual (left, $588 \mathrm{vs} 578 \mathrm{~ms}, p=$ 0.15 ) and auditory (right, $491 \mathrm{vs} 476 \mathrm{~ms}, p=0.01$ ) decisions.

$p=0.013$; effects were individually significant in 6 of 11 sites; $p<$ $0.01, t$ test $)$.

Altogether, the probabilistic choice analysis suggests that the reduced decision accuracy on visual trials was largely driven by a consistent, reduced sensitivity to visual inputs that was sometimes exacerbated by a bias away from contralateral choices.

A final potential explanation for the reduced visual decision accuracy (Fig. $3 A$ ) is that the animals accumulated evidence for less time on stimulation versus control trials. This could reduce visual decision accuracy even if sensitivity were unchanged: a shorter accumulation time would lead the animal to miss evidence. To gain insight into accumulation time, we measured the degree to which instantaneous fluctuations in stimulus rate influenced the rat's eventual decision (see Materials and Methods). This analysis demonstrated that, on both stimulation and control trials, evidence presented throughout the trial influenced the animal's eventual decision (Fig. $4 D$, blue and black lines $>0$ for the entire trial). This is consistent with long accumulation times for both stimulation and control trials and argues against the possibility that stimulation affected the duration over which evidence was accumulated. Notably, evidence at each moment had a weaker influence on stimulation versus control trials (Fig. $4 D$, blue line below black line). This difference was significant ( $p=$ $0.0001, t$ test) and is consistent with the loss of sensitivity indicated by the probabilistic choice model.

To ensure that the effects observed were due to ChR2 activation, we repeated the same stimulation protocol in a rat not injected with ChR2 (Fig. 5A). Similar values were observed on stimulation and control trials for bias ( $p=0.22, t$ test) and sensitivity ( $p=0.20, t$ test). This indicates that blue light in the brain does not by itself drive the effects that we observed.

Together, these analyses provide strong evidence that PPC disruption reduced the rat's sensitivity on visual decisions.

\section{Optogenetic disruption of PPC largely spared auditory decision making}

We evaluated performance on interleaved auditory trials to determine whether the effects reported so far reflected a loss of sensitivity that was specific to vision or instead reflected more general decision-making deficits. Auditory decisions from the same site and sessions as in Figure $4 \mathrm{~A}$ demonstrate a much weaker effect of stimulation (Fig. $4 G$ ). Some sites (4/11) did have small reductions in sensitivity that reached significance $(p<$ 0.01; Fig. $4 H$, points below dashed line). Across sites, however, this reduction in sensitivity was not significant (Fig. $4 H, p=$ 0.13 ). Further, a site-by-site comparison revealed that visual sensitivity was significantly more reduced by stimulation compared with auditory sensitivity (Fig. $6 A ; p=0.0021, t$ test).

No consistent effect was observed on animals' reliance on trial history regardless of whether it was a previous trial's success (Fig. $4 I, p=0.16, t$ test) or failure (Fig. $4 K, p=0.28, t$ test).

The effect on bias was idiosyncratic. As with visual trials, an ipsilateral bias was sometimes present, but biases in the opposite direction were also observed [Fig. $4 L$; a significant ipsilateral bias (same direction as for visual trials) was evident at 6 of 10 sites and a significant contralateral bias was evident at 2 of 10 individual sites, $p<$ $0.01, t$ test]. No significant change was present overall (Fig. $4 L$, $p=$ $0.13, t$ test). The difference in bias between auditory and visual trials did not reach significance (Fig. $6 B, p=0.29, t$ test). Heterogeneity in bias effects may reflect that there are two or more subgroups of neurons in PPC with distinct projection targets (Li et al., 2015).

We repeated the logistic regression analysis, examining the effects of previous left and right decisions separately. We still failed to find consistent evidence that stimulation changed the animal's strategy, although there were two significant cases: First, animals tended to repeat successful visual, contralateral choices significantly more on stimulation versus control trials $(p=$ $0.0057)$. This was not the case for successful auditory contralateral choices ( $p=0.13)$. Second, animals tended to repeat failed auditory, contralateral choices significantly more on stimulation versus control trials $(p=0.013)$. This was not the case for failed visual contralateral choices $(p=0.074)$. This more specific analysis of trial history reinforces our finding that the effect of previous choice is not affected consistently by PPC disruption.

Finally, as with visual decisions, we measured the degree to which instantaneous fluctuations in auditory stimulus rate influenced the rat's eventual decision (see Materials and Methods). Again, we found that, on both stimulation and control trials, evidence presented throughout the trial influenced the animal's eventual decision (Fig. 4J, blue and black lines $>0$ for the entire 

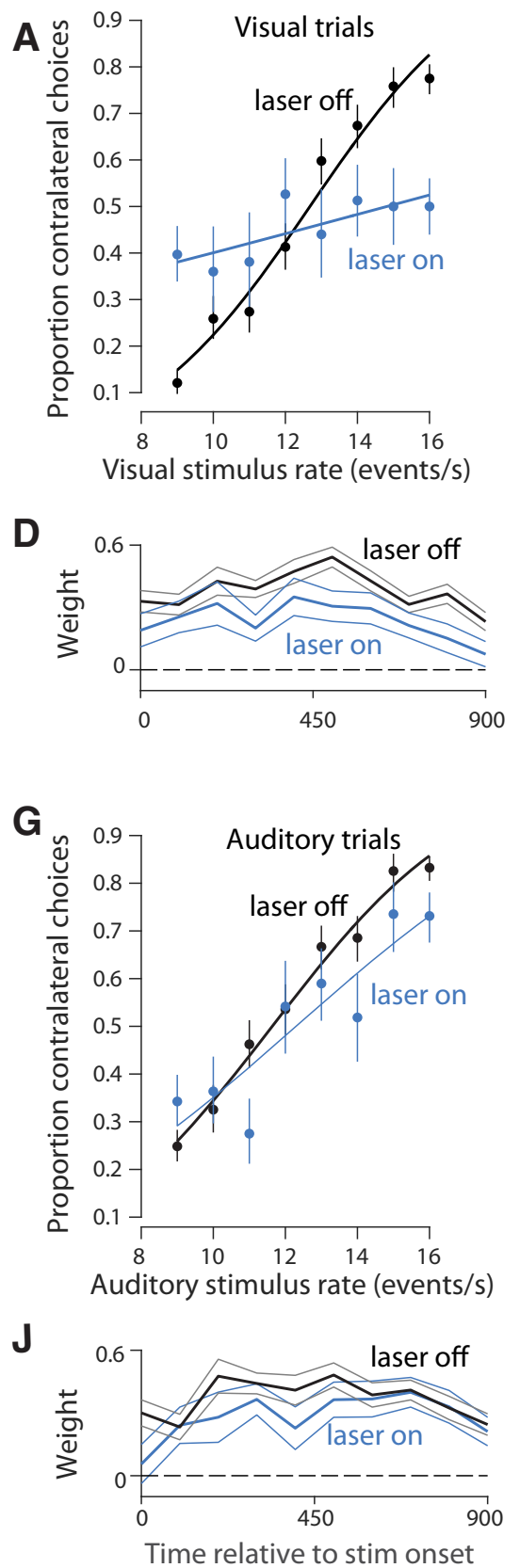
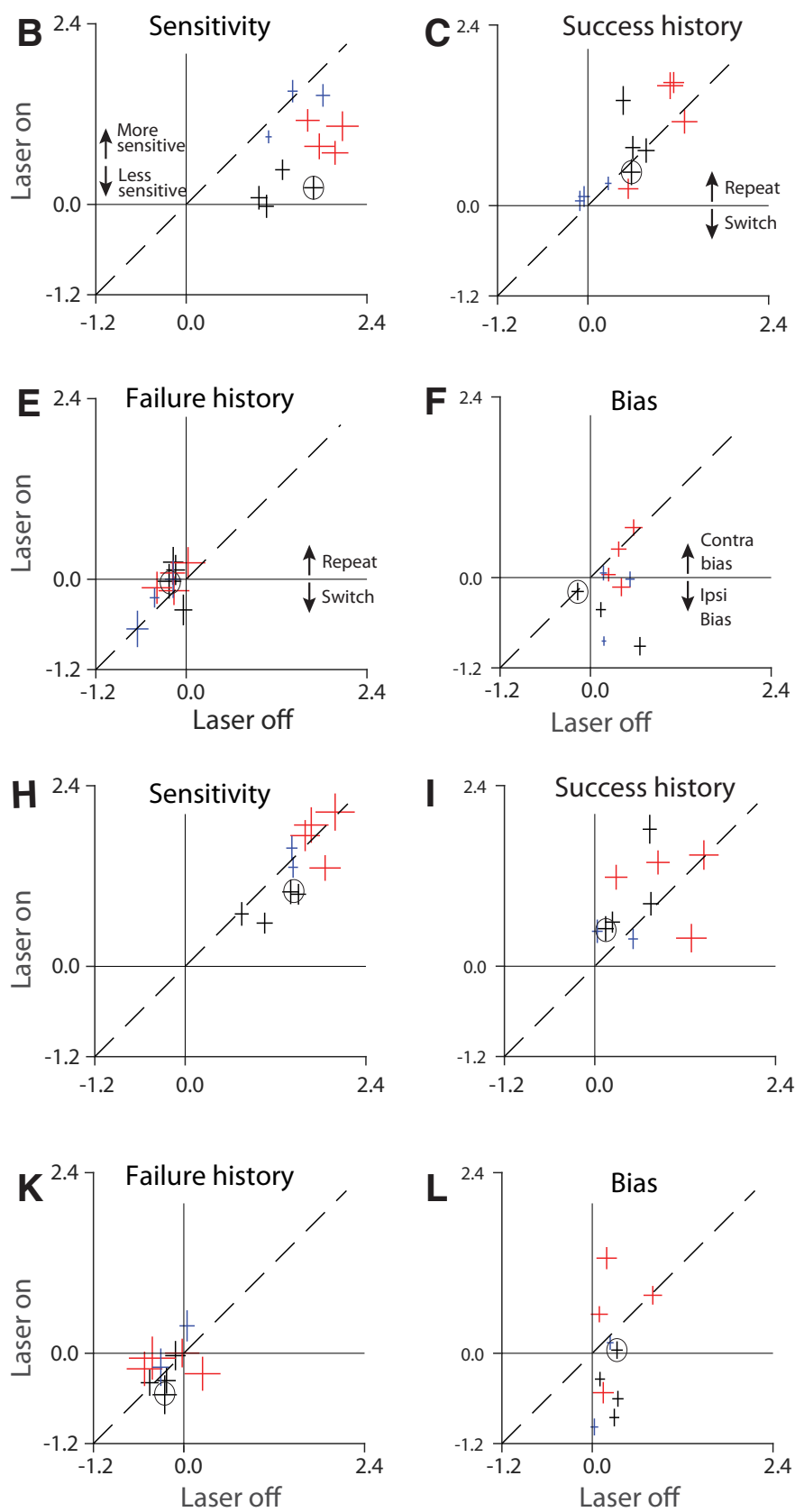

Figure 4. PPC disruption has a larger effect on visual, compared with auditory, decisions. $A$, Visual psychometric functions from stimulation in a single location within PPC (Rat 1,1624 trials). Smooth lines are fits to the data (logistic regression). Error bars reflect the Wilson binomial confidence interval. $\boldsymbol{B}$, Outcome of a probabilistic model that measures the effect of sensitivity to stimulus rate on decisions. The fitted parameter is plotted for stimulation (laser-on, vertical axis) versus control (laser-off, horizontal axis) trials. All values are positive, indicating that increasing stimulus rate led to more high rate decisions. Error bars indicate SEs. Dashed line, $y=x$. Colors: individual rats; multiple points for each animal indicate data collected from different optical fibers/depths (sites) within PPC. Black circle indicates the animal shown in $\boldsymbol{A}$. C, Same as $\boldsymbol{B}$ but for the "success history" parameter. Positive values indicate that the rat tended to repeat rewarded decisions. $\boldsymbol{D}$, Weighting of visual sensory evidence during $100 \mathrm{~ms}$ windows. Vertical axis indicates the weight for the time indicated by the corresponding value on the horizontal axis. Weights were computed via logistic regression (see Materials and Methods). Values would approximate dashed line ( $y=0$ ) for times during which sensory evidence failed to influence the choice. Black line indicates control (15,267 trials). Cyan line: stimulation ( 6046 trials). $\boldsymbol{E}$, Same as $\boldsymbol{B}$ but for the "failure history" parameter. Negative values indicate the rat tended to switch after unrewarded decisions. $\boldsymbol{F}$, Same as $\boldsymbol{B}$ but for the "bias" parameter. Zero indicates unbiased decisions; negative values indicate an ipsilateral bias. $\mathbf{G}$, Auditory psychometric functions from the same rat/site as in $(\boldsymbol{A})$ ( 1655 trials). $\boldsymbol{H}, \boldsymbol{I}, \boldsymbol{K}, \boldsymbol{L}$, Same as $\boldsymbol{B}, \boldsymbol{C}, \boldsymbol{E}, \boldsymbol{F}$, respectively, but for auditory trials. J, Same as $\boldsymbol{D}$ but for auditory trials. Black line indicates control (10,148 trials). Cyan line indicates stimulation (4665 trials).

trial). This argues against the possibility that stimulation affected the duration over which evidence was accumulated. Weights were slightly reduced on stimulation compared with control trials $(p=0.02)$, again consistent with a weaker and inconsistent effect on auditory sensitivity.

Together, these analyses provide strong evidence that PPC disruption had only a weak effect on the rat's sensitivity on auditory decisions.

\section{PPC neurons are more strongly driven by individual visual} events than by auditory events

The consistent effect of stimulation on visual, but not auditory, sensitivity argues against a simple model in which auditory and visual signals influence PPC equally (Fig. 7A). Our results suggest a new class of model in which PPC is a key player for translating visual, but not auditory, sensory signals into decisions (Fig. $7 B, C)$. To provide an independent test of this class of model, we 
A

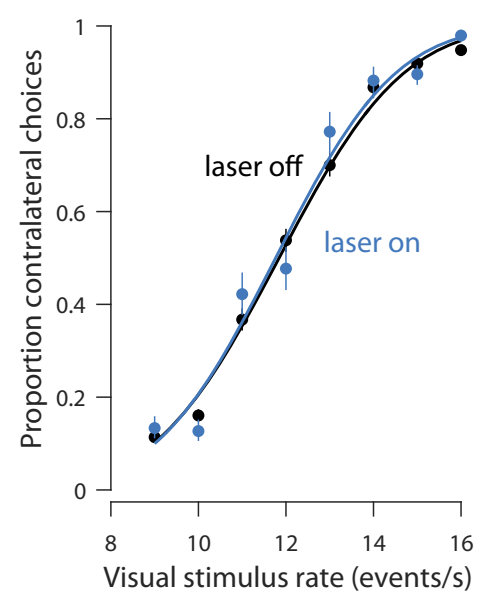

B

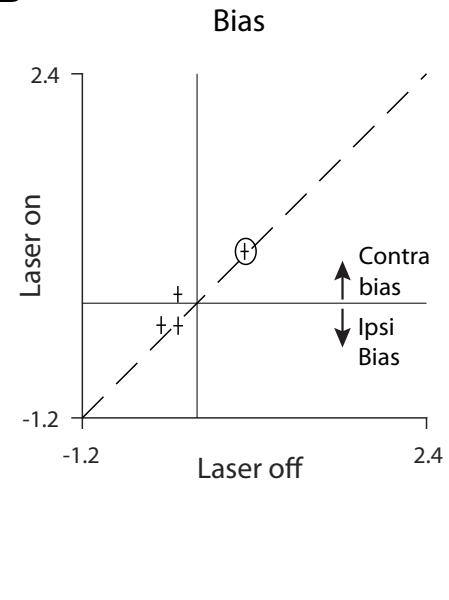

C

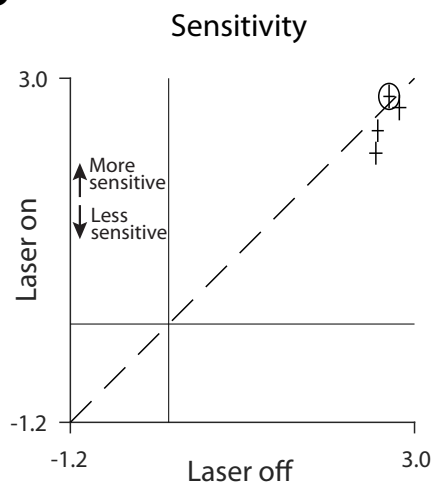

Figure 5. In an uninjected rat, decisions are unaffected by blue light. $A$, Psychometric functions for sessions in which a single laser was used and black tape was applied to prevent light from escaping the sides of the implanted chamber (5781 trials). No effect was seen on choice $(p=0.33)$ or sensitivity $(p=0.31)$. $\boldsymbol{B}, \boldsymbol{C}$, Effects on bias and sensitivity from four experiments (same conventions as in Fig. $4 B, F$ ) for the animal in $\boldsymbol{A}$. The site in $A$ is indicated by the circled points. Solid lines indicate 0 .

evaluated whether individual visual sensory events had a larger impact on PPC responses compared with individual auditory sensory events using a previously collected, large-scale ( $N=101,972$ successful trials) electrophysiological dataset (Raposo et al., 2014). This sensory-evoked response is potentially separate from the decisionrelated responses reported in previous analyses (Raposo et al., 2014), which focused on slower signals evolving over an entire $1000 \mathrm{~ms}$ decision (Fig. 8A) rather than on transient responses after individual sensory events. Indeed, a signature of individual events can be obscured when trials with events at different times are averaged, especially when the slower decision modulation is large (Fig. 8A) or with wide-filter smoothing (as is often used to improve signal-to-noise ratios). We evaluated the impact of individual auditory and visual events by aligning electrophysiological responses to individual visual or auditory events in single neurons and removing the slow component (see Materials and Methods). Many neurons were driven by individual stimulus events (Fig. $8 B$ ). This event modulation was frequently evident in visual trials ( 84 of 317 neurons at $p<0.01$ ), but only occasionally in auditory trials ( 5 of 317 neurons at $p<0.01$ ). Modulation was significantly more common due to visual compared with auditory events $\left(p \ll 10^{-4}, \chi^{2} 2 \times 2\right.$ contingency table). Modulation was also significantly stronger for visual compared with auditory events within neurons (Fig. $8 C, D)$. This greater modulation for visual events was significant in $4 / 5$ individual animals (paired sign test, $p \ll 10^{-4} ; p=$ $0.50 ; p=0.0008 ; p=0.0003 ; p=0.035$.
A

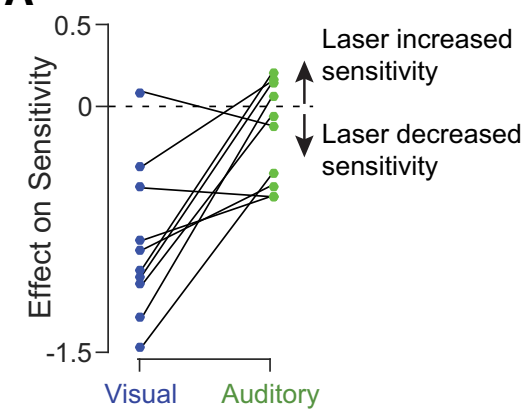

Figure 6. Site-by-site comparison of stimulation effects on bias and sensitivity for auditory and visual trials. Each point was computed by taking the difference in the regression coefficients between stimulation and control trials for each modality at each stimulation site in the three rats. Coefficients obtained from auditory and visual trials at the same stimulation site (interleaved trials) are connected with a solid line. Dashed line at 0 indicates no effect of stimulation. $\boldsymbol{A}$, Stimulation caused a larger reduction on visual sensitivity (blue points) compared with auditory sensitivity (green points). This difference was significant ( $t$ test, $p=$ 0.0021). $\boldsymbol{B}$, Stimulation caused a larger ipsilateral bias on visual (blue) compared with auditory (green) decisions. However, this difference did not reach significance $(t$ test, $p=0.29)$.

A



B

Local accumulation model

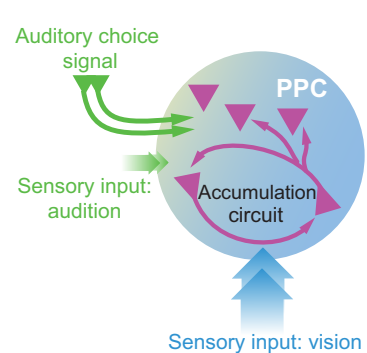

C

Remote accumulation model

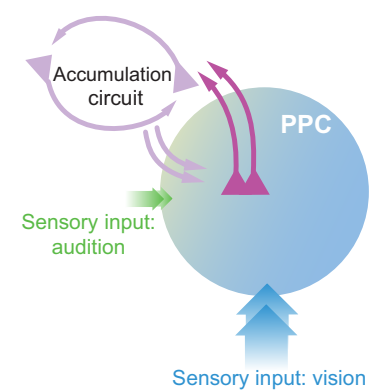

Figure 7. Putative models for PPC's role in a decision circuit. $A$, Balanced input model that is unsupported by the disruption experiment. $\boldsymbol{B}$, Local accumulation model in which visual inputs to PPC are stronger than auditory inputs and evidence over time is accumulated within PPC. Visual choice signals are therefore computed locally within PPC as the output of the accumulation circuit (pink). Auditory information is accumulated elsewhere and fed back to PPC (green arrows). C, Remote accumulation model in which visual inputs to PPC are stronger than auditory inputs and evidence over time is accumulated at a remote location and fed back to PPC. 
A

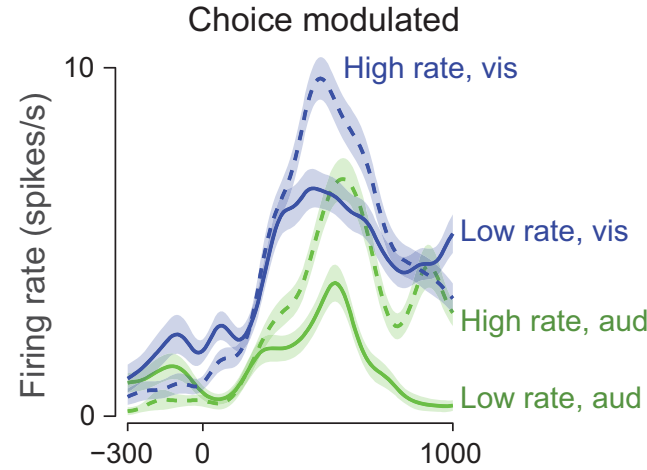

Time from sensory stim. on (ms)
B

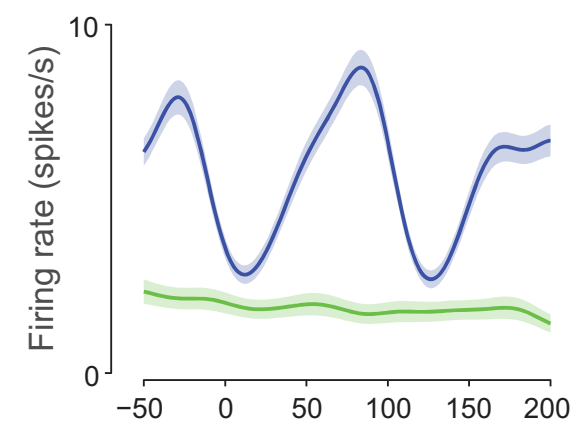

Time from sensory event (ms)
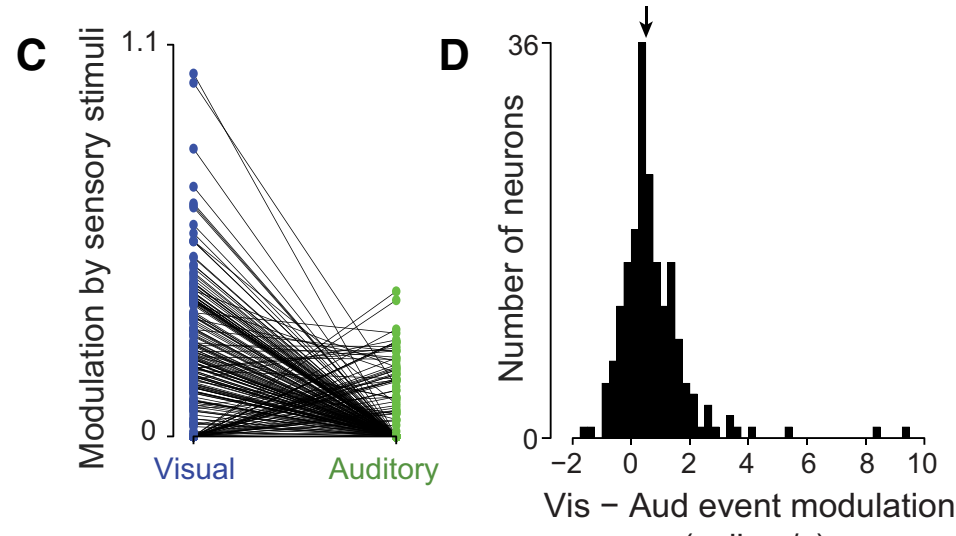

$\mathbf{E}$

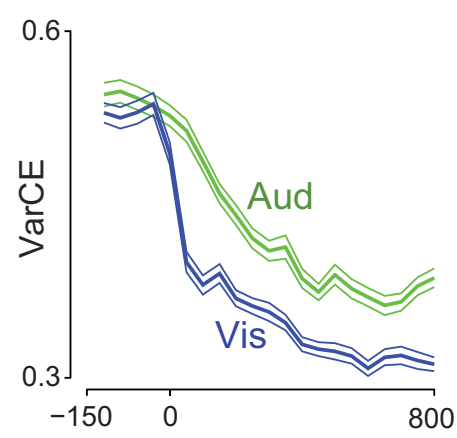

Time from sensory stim. on (ms)

Figure 8. Electrophysiological analyses suggesting that PPC discriminates individual visual events and does not act as an evidence accumulator. $\boldsymbol{A}$, Trial-averaged peristimulus time histogram for an example neuron. Solid traces indicate low-rate trials; dashed traces, high-rate trials; blue traces, visual trials; and green traces, auditory trials. Transparent fills show SEM. Outcome of the decision (dashed vs solid lines) and the stimulus modality (blue vs green lines) drove slow modulations over the 1000 ms decision. $\boldsymbol{B}$, PETH for same neuron as in $\boldsymbol{A}$ aligned to individual visual or auditory events (see Materials and Methods). Same color conventions as $\boldsymbol{A}$; only responses to low rate trials are shown. $\boldsymbol{C}$, Modulation strength of each neuron by visual (blue) and auditory (green) events. Vertical axis indicates spikes per second plotted on a log scale. Data are aggregated across five animals. The two measurements for each neuron are connected by a line. Note that, after correcting for noise, many neurons had a modulation index of 0 . D, Histogram over neurons of the modulation index for visual minus the index for auditory (same dataset as in $\boldsymbol{C}$ ). Neurons with both modulation indices equal to 0 were excluded. Arrow shows median $\left(0.68 ; p<10^{-10}\right.$, sign test). $\boldsymbol{E}$, VarCE computed relative to stimulus onset for auditory (green) and visual (blue) trials.

Importantly, a larger effect of visual inputs was evident despite the fact that auditory and visual stimuli were carefully matched so that they had an equivalent effect on decisions (see Fig. $1 C$ of Raposo et al., 2014). These observations, like the disruption effects, support models in which visual inputs are dominant in PPC (Fig. $7 B, C$ ).

\section{Analysis of trial-to-trial variability suggests sensory signals are accumulated remotely}

Visual inputs to PPC might be accumulated locally within the area (local accumulation model; Fig. $7 B$ ) or might be accumulated in another region that projects back to PPC (remote accumulation model; Fig. 7C). Theory suggests that, as integrators accumulate evidence, they should also accumulate noise that will vary across trials (Churchland et al., 2011). A rise in VarCE that is at least transient is predicted for any model that strongly includes accumulating evidence or count in some way. This is because the early evidence in some trials points toward one decision, whereas the early evidence in other trials points toward the opposite decision.

Therefore, trial-to-trial variability should increase during decision formation in areas that reflect evidence accumulation. In contrast, in areas dominated by sensory inputs or movement preparation, the stabilizing influence of the input or motor plan will drive decreases in trial-to-trial variability over time in a trial
(Churchland et al., 2010; Rajan et al., 2010). A measure of trialto-trial variability in neural responses, the VarCE (Churchland et al., 2011), was designed to distinguish these possibilities. In several decision-related brain areas in the monkey, the VarCE has been demonstrated to increase over time during evidence accumulation decisions (Churchland et al., 2011; Ding, 2015). In the present data, clear decreases in VarCE were observed for both auditory and visual trials (Fig. $8 E$ ).

\section{Discussion}

Our results argue that PPC plays a causal role in the sensory processing aspects of visual decision making and that choice signals that have been observed electrophysiologically may reflect computations that take place elsewhere in the circuit. Three observations support this. First, optogenetic disruption of PPC reduces sensitivity on visual decisions but largely spares auditory decisions and does not affect movement metrics or the time course of evidence accumulation (Figs. 3, 4). Second, in our task, individual visual events drive larger electrophysiological responses in single neurons compared with auditory events (Fig. $8 B-D)$, even though these events are equally effective in driving behavior. Finally, trial-to-trial variability decreases during decisions, suggesting the presence of an overall stabilizing influence from sensory inputs or action planning rather than the destabilizing influence of evidence accumulation (Fig. 8E). Together, 
these findings point to PPC as required to process and perhaps discriminate visual signals that are then accumulated remotely (Fig. 7C).

\section{Understanding with precision the effects of PPC disruption on behavior}

The experimental design here allowed us to go far beyond previous disruption studies because we used temporally precise disruption, included multiple sensory modalities, and analyzed the decisions with in-depth behavioral analyses. This approach allowed us to understand the nature of the deficit with precision and revealed a specific loss of sensitivity on visual decisions. This deficit could have arisen because PPC is needed in this task to discriminate individual sensory events for subsequent accumulation, perhaps pooling information from V1 neurons with the appropriate temporal frequency tuning or even reformatting the information for a linear decoder (Rust and Dicarlo, 2010). Alternatively, PPC may be required to support the attentional requirements of the demanding task that we used in this study. A role in attention and prioritizing space has long been attributed to PPC in primates (for reviews, see Colby and Goldberg, 1999; Gottlieb, 2007). Having established that PPC plays a causal role in visual decisions, future studies should aim to uncover the computations performed in PPC that support these decisions.

Our results offer a unifying explanation for why inactivation during auditory decisions sometimes has little effect (Erlich et al., 2015) despite strong modulation of PPC neurons during such decisions (Hanks et al., 2015). Specifically, the evidence accumulation (Hanks et al., 2015) and choice (Raposo et al., 2014) signals present in PPC electrophysiological recordings may reflect feedback from other areas (Fig. 7C). This feedback possibility has been raised previously and receives direct support here by our measure of trial-to-trial variability, the VarCE, which is diagnostic of underlying neural computations. The VarCE will increase in areas that reflect accumulation of evidence using any of a number of strategies because some amount of noise will accumulate with the signal (Churchland et al., 2011). A rising VarCE has been observed in primate lateral intraparietal area (Churchland et al., 2011) and caudate nucleus and frontal eye field (Ding, 2015). In contrast, VarCE decreases in areas that reflect sensory input or action planning (Churchland et al., 2010) because those computations push the network toward a more stabilized state (Rajan et al., 2010). The decreasing VarCE observed here may likewise indicate stabilization. Future studies will be needed to determine whether this stabilization is driven by sensory input, action planning, or both. We speculate that the sharper decrease in the VarCE seen on visual decisions (Fig. 8E, blue) may reflect the dual stabilizing influences of visual sensory input and action planning feedback, whereas the slower and less deep decrease of the VarCE on auditory decisions (Fig. 8E, green) may reflect only a single stabilizing influence, most likely action-planning feedback. This difference highlights the important possibility that neurons might be heterogeneous in the extent to which they tend to stabilize versus destabilize the network over time. Future work with identified cell types will be powerful in understanding heterogeneity across neurons in this regard. In any event, the idea that PPC neurons reflect, in part, action-planning signals is consistent with previous observations that the direction and magnitude of decision-related tuning does not depend strongly on whether decisions were instructed by auditory or visual inputs (Raposo et al., 2014).

\section{Challenges in interpreting neural disruption experiments}

Disrupting neural activity to determine a structure's role in behavior, as we have done here, can lead to challenges in interpretation (Otchy et al., 2015). Fortunately, a number of aspects of our experimental design bolstered our ability to interpret these disruption experiments. First, we minimized the chance that the rats would detect the disruption and adjust their strategy: the disruption was transient and present only on a minority of randomly selected trials. This allowed us to rule out that the sparing of auditory decisions was explained by reliance on alternate circuits during slow time course inactivation, a possibility left open by other studies of decisions (Raposo et al., 2014; Erlich et al., 2015; Katz et al., 2016). Second, we disrupted activity by artificially elevating firing rates, a method that is suitable for disruption of behaviors that depend on heterogeneous and timevarying population codes (Churchland and Shenoy, 2007; Roberts et al., 2012; Rodgers and DeWeese, 2014; Otchy et al., 2015). For such behaviors, optogenetic stimulation offers some advantages over optogenetic suppression because it introduces a new, aberrant signal. This may more strongly perturb the population code compared with suppression, especially because the overall change to the population can be larger than for suppression (which suffers floor effects).

A final aspect of our experimental design that aids interpretation of effects is that we studied decisions guided by two different sensory modalities. This allowed us to rule out some alternatives to the possibility that PPC disruption reduces visual sensitivity. For instance, one alternative explanation for the deficits during visual decisions is that PPC stimulation altered decision making by activating neurons in downstream circuits that plan the actions needed for decision reporting. We can rule out these actionplanning "off-target" effects (Otchy et al., 2015) because auditory decisions, which would rely on the same motor circuits, were largely spared. Other off-target effects are harder to rule out and even interpret. For instance, if PPC has denser feedback projections to primary visual cortex than to primary auditory cortex, then PPC stimulation might have stronger effects on primary visual cortex neurons, explaining the largely visual deficits that we observed. However, our optogenetic disruptions produced similar behavioral effects as previously reported inactivations (Raposo et al., 2014), making it unlikely that these effects were solely caused by the very different mechanisms of disruption via feedback. Regardless, an independent support for a role of PPC in discriminating visual events is provided by our observation from electrophysiology that visual inputs more strongly drive the temporally precise PPC responses that are needed to discriminate visual inputs.

An additional caveat is that the extent of the disruption due to direct activation is not known with absolute precision. This is because, although we measured neural activity during stimulation, the spatial coverage of our electrodes was insufficient to determine at what distance from the stimulating electrode the blue light ceased to activate neurons. Fortunately, for optogenetic disruption, the spatial extent of activation is primarily determined by parameters of the stimulation: wavelength, fiber diameter, numerical aperture, and laser power. This is unlike chemogenetic inactivation, in which the spatial extent of activation depends on the spread of viral infection, or pharmacological disruption, in which the spatial extent of activation depends on diffusion of the reagent. To estimate light spread, and thus the spatial extent of our disruption, we used published calculators (see Materials and Methods).

Although we think it unlikely that our results were due to direct stimulation of V1 neurons (see Materials and Methods), it is essential to acknowledge that outstanding questions remain in 
understanding the relationship between PPC, classically defined by its thalamic inputs (Chandler et al., 1992; Reep et al., 1994), and the secondary visual areas that are observed via anatomical tracing (Coogan and Burkhalter, 1990; Montero, 1993). The shallower psychometric functions that we observed on stimulation trials (Fig. $4 A$ ) are reminiscent of those seen during inactivation of extrastriate regions in monkey (see Fig. 2C of Katz et al., 2016). One possibility is that rat PPC shares features with monkey extrastriate regions, such as a causal role in processing raw visual inputs (Newsome and Paré, 1988; Katz et al., 2016). If so, future experiments are needed to establish which stimuli are effective in eliciting responses (both visual and other modalities), and the extent to which this depends on behavioral context. These experiments would bolster the observations that we made here about the impact of individual auditory and visual events. Indeed, recent work (Song et al., 2017) emphasizes the importance of context: in the context of a Go/No-Go conflict task, in which the precision of cue timing may influence behavior, mouse PPC may be more influenced by auditory stimuli than we observed in our stimulus-congruent, temporal integration task.

An alternative to the possibility that rat PPC has features in common with monkey extrastriate cortex is that rat PPC may be akin to monkey PPC (Brody and Hanks, 2016) and the extrastriate-like deficits that we observed are present because the PPC coordinates used by us and others (Whitlock et al., 2012; Raposo et al., 2014; Erlich et al., 2015) encompass separate, more extrastriate-like areas. Challenges in distinguishing a candidate structure from its nearby neighbors have long been acknowledged. The present results make clear that at least some of this cortical territory is causally involved in visual decision making. However, improved resolution of areas and their borders using methods such as wide-field retinotopic mapping (Schuett et al., 2002; Andermann et al., 2011; Garrett et al., 2014; Glickfeld et al., 2014) and noise analyses (Kiani et al., 2015) combined with highdensity recordings may inform further experiments narrowing down the key areas for decision making in cortex.

\section{Role for PPC in visual processing}

In conclusion, we demonstrate that PPC plays a causal role specifically in visual decision making. Our results are consistent with previous inactivation studies, but allowed us to probe more deeply the effects of disruption by ruling out alternative explanations for the deficits to visual decision making. Further, our analysis of electrophysiological responses provides independent evidence of a dominant role for vision in PPC. By establishing PPC as part of a circuit for visual decision making, we pave the way for future studies that will reveal how visual signals within PPC are transformed as they are passed to subsequent areas.

\section{References}

Andermann ML, Kerlin AM, Roumis DK, Glickfeld LL, Reid RC (2011) Functional specialization of mouse higher visual cortical areas. Neuron 72:1025-1039. CrossRef Medline

Anikeeva P, Andalman AS, Witten I, Warden M, Goshen I, Grosenick L, Gunaydin LA, Frank LM, Deisseroth K (2011) Optetrode: a multichannel readout for optogenetic control in freely moving mice. Nat Neurosci 15:163-170. CrossRef Medline

Aravanis AM, Wang LP, Zhang F, Meltzer LA, Mogri MZ, Schneider MB, Deisseroth K (2007) An optical neural interface: in vivo control of rodent motor cortex with integrated fiberoptic and optogenetic technology. J Neural Eng 4:S143-156. CrossRef Medline

Boyden ES, Zhang F, Bamberg E, Nagel G, Deisseroth K (2005) Millisecondtimescale, genetically targeted optical control of neural activity. Nat Neurosci 8:1263-1268. CrossRef Medline
Brody CD, Hanks TD (2016) Neural underpinnings of the evidence accumulator. Curr Opin Neurobiol 37:149-157. CrossRef Medline

Brostek L, Büttner U, Mustari MJ, Glasauer S (2013) Neuronal variability of MSTd neurons changes differentially with eye movement and visually related variables. Cereb Cortex 23:1774-1783. CrossRef Medline

Busse L, Ayaz A, Dhruv NT, Katzner S, Saleem AB, Schölvinck ML, Zaharia $A D$, Carandini M (2011) The detection of visual contrast in the behaving mouse. J Neurosci 31:11351-11361. CrossRef Medline

Chandler HC, King V, Corwin JV, Reep RL (1992) Thalamocortical connections of rat posterior parietal cortex. Neurosci Lett 143:237-242. CrossRef Medline

Chen A, Gu Y, Liu S, DeAngelis GC, Angelaki DE (2016) Evidence for a causal contribution of macaque vestibular, but not intraparietal, cortex to heading perception. J Neurosci 36:3789-3798. CrossRef Medline

Churchland AK, Kiani R, Shadlen MN (2008) Decision-making with multiple alternatives. Nat Neurosci 11:693-702. CrossRef Medline

Churchland AK, Kiani R, Chaudhuri R, Wang XJ, Pouget A, Shadlen MN (2011) Variance as a signature of neural computations during decision making. Neuron 69:818-831. CrossRef Medline

Churchland MM et al. (2010) Stimulus onset quenches neural variability: a widespread cortical phenomenon. Nat Neurosci 13:369-378. CrossRef Medline

Churchland MM, Shenoy KV (2007) Delay of movement caused by disruption of cortical preparatory activity. J Neurophysiol 97:348-359. CrossRef Medline

Colby CL, Goldberg ME (1999) Space and attention in parietal cortex. Annu Rev Neurosci 22:319-349. CrossRef Medline

Coogan TA, Burkhalter A (1990) Conserved patterns of cortico-cortical connections define areal hierarchy in rat visual cortex. Exp Brain Res 80:49-53. Medline

Crowne DP, Richardson CM, Dawson KA (1986) Parietal and frontal eye field neglect in the rat. Behav Brain Res 22:227-231. CrossRef Medline

Ding L (2015) Distinct dynamics of ramping activity in the frontal cortex and caudate nucleus in monkeys. J Neurophysiol 114:1850-1861. CrossRef Medline

Ding L, Gold JI (2012) Separate, causal roles of the caudate in saccadic choice and execution in a perceptual decision task. Neuron 75:865-874. CrossRef Medline

Erlich JC, Brunton BW, Duan CA, Hanks TD, Brody CD (2015) Distinct effects of prefrontal and parietal cortex inactivations on an accumulation of evidence task in the rat. eLife 4.

Garrett ME, Nauhaus I, Marshel JH, Callaway EM (2014) Topography and areal organization of mouse visual cortex. J Neurosci 34:12587-12600. CrossRef Medline

Geisler WS, Albrecht DG (1995) Bayesian analysis of identification performance in monkey visual cortex: nonlinear mechanisms and stimulus certainty. Vision Res 35:2723-2730. CrossRef Medline

Glickfeld LL, Reid RC, Andermann ML (2014) A mouse model of higher visual cortical function. Curr Opin Neurobiol 24:28-33. CrossRef Medline

Gold JI, Ding L (2013) How mechanisms of perceptual decision-making affect the psychometric function. Prog Neurobiol 103:98-114. CrossRef Medline

Gottlieb J (2007) From thought to action: the parietal cortex as a bridge between perception, action, and cognition. Neuron 53:9-16. CrossRef Medline

Guo ZV, Li N, Huber D, Ophir E, Gutnisky D, Ting JT, Feng G, Svoboda K (2014) Flow of cortical activity underlying a tactile decision in mice. Neuron 81:179-194. CrossRef Medline

Hanks TD, Ditterich J, Shadlen MN (2006) Microstimulation of macaque area LIP affects decision-making in a motion discrimination task. Nat Neurosci 9:682-689. CrossRef Medline

Hanks TD, Kopec CD, Brunton BW, Duan CA, Erlich JC, Brody CD (2015) Distinct relationships of parietal and prefrontal cortices to evidence accumulation. Nature 520:220-223. CrossRef Medline

Harvey CD, Coen P, Tank DW (2012) Choice-specific sequences in parietal cortex during a virtual-navigation decision task. Nature 484:62-68. CrossRef Medline

Huber D, Petreanu L, Ghitani N, Ranade S, Hromádka T, Mainen Z, Svoboda K (2008) Sparse optical microstimulation in barrel cortex drives learned behaviour in freely moving mice. Nature 451:61-64. CrossRef Medline Katz LN, Yates JL, Pillow JW, Huk AC (2016) Dissociated functional signif- 
icance of decision-related activity in the primate dorsal stream. Nature 535:285-288. CrossRef Medline

Kiani R, Cueva CJ, Reppas JB, Peixoto D, Ryu SI, Newsome WT (2015) Natural grouping of neural responses reveals spatially segregated clusters in prearcuate cortex. Neuron 85:1359-1373. CrossRef Medline

Lau B, Glimcher PW (2005) Dynamic response-by-response models of matching behavior in rhesus monkeys. J Exp Anal Behav 84:555-579. CrossRef Medline

Li N, Chen TW, Guo ZV, Gerfen CR, Svoboda K (2015) A motor cortex circuit for motor planning and movement. Nature 519:51-56. CrossRef Medline

Marcos E, Pani P, Brunamonti E, Deco G, Ferraina S, Verschure P (2013) Neural variability in premotor cortex is modulated by trial history and predicts behavioral performance. Neuron 78:249-255. CrossRef Medline

Montero VM (1993) Retinotopy of cortical connections between the striate cortex and extrastriate visual areas in the rat. Exp Brain Res 94:1-15. Medline

Nawrot MP, Boucsein C, Rodriguez Molina V, Riehle A, Aertsen A, Rotter S (2008) Measurement of variability dynamics in cortical spike trains. J Neurosci Methods 169:374-390. CrossRef Medline

Newsome WT, Paré EB (1988) A selective impairment of motion perception following lesions of the middle temporal visual area (MT). J Neurosci 8:2201-2211. Medline

Otchy TM, Wolff SB, Rhee JY, Pehlevan C, Kawai R, Kempf A, Gobes SM, Ölveczky BP (2015) Acute off-target effects of neural circuit manipulations. Nature 528:358-363. CrossRef Medline

Paxinos G, Watson C (2007) The rat brain in stereotaxic coordinates, Ed 5. Amsterdam; Boston: Academic/Elsevier.

Rajan K, Abbott LF, Sompolinsky H (2010) Stimulus-dependent suppression of chaos in recurrent neural networks. Phys Rev E Stat Nonlin Soft Matter Phys 82:011903. CrossRef Medline

Raposo D, Sheppard JP, Schrater PR, Churchland AK (2012) Multisensory decision-making in rats and humans. J Neurosci 32:3726-3735. CrossRef Medline

Raposo D, Kaufman MT, Churchland AK (2014) A category-free neural population supports evolving demands during decision-making. Nat Neurosci 17:1784-1792. CrossRef Medline

Reep RL, Chandler HC, King V, Corwin JV (1994) Rat posterior parietal cortex: topography of corticocortical and thalamic connections. Exp Brain Res 100:67-84. CrossRef Medline

Rishel CA, Huang G, Freedman DJ (2013) Independent category and spatial encoding in parietal cortex. Neuron 77:969-979. CrossRef Medline

Roberts TF, Gobes SM, Murugan M, Ölveczky BP, Mooney R (2012) Motor circuits are required to encode a sensory model for imitative learning. Nat Neurosci 15:1454-1459. CrossRef Medline

Rodgers CC, DeWeese MR (2014) Neural correlates of task switching in prefrontal cortex and primary auditory cortex in a novel stimulus selection task for rodents. Neuron 82:1157-1170. CrossRef Medline

Rogan SC, Roth BL (2011) Remote control of neuronal signaling. Pharmacol Rev 63:291-315. CrossRef Medline

Roitman JD, Shadlen MN (2002) Response of neurons in the lateral intraparietal area during a combined visual discrimination reaction time task. J Neurosci 22:9475-9489. Medline

Rust NC, Dicarlo JJ (2010) Selectivity and tolerance ("invariance") both increase as visual information propagates from cortical area V4 to IT. J Neurosci 30:12978-12995. CrossRef Medline

Schuett S, Bonhoeffer T, Hübener M (2002) Mapping retinotopic structure in mouse visual cortex with optical imaging. J Neurosci 22:6549-6559. Medline

Shadlen MN, Newsome WT (1998) The variable discharge of cortical neurons: implications for connectivity, computation, and information coding. J Neurosci 18:3870-3896. Medline

Sheppard JP, Raposo D, Churchland AK (2013) Dynamic weighting of multisensory stimuli shapes decision-making in rats and humans. J Vis 13.

Song YH, Kim JH, Jeong HW, Choi I, Jeong D, Kim K, Lee SH (2017) A neural circuit for auditory dominance over visual perception. Neuron 93:1236-1237. CrossRef Medline

Suzuki M, Gottlieb J (2013) Distinct neural mechanisms of distractor suppression in the frontal and parietal lobe. Nat Neurosci 16:98-104. CrossRef Medline

Whitlock JR, Pfuhl G, Dagslott N, Moser MB, Moser EI (2012) Functional split between parietal and entorhinal cortices in the rat. Neuron 73:789 802. CrossRef Medline

Znamenskiy P, Zador AM (2013) Corticostriatal neurons in auditory cortex drive decisions during auditory discrimination. Nature 497:482-485. CrossRef Medline 